\title{
Sub-inertial modulation of nonlinear Kelvin waves in the coastal zone
}

\author{
D. V. Stepanov ${ }^{1,2}$ and V. V. Novotryasov ${ }^{1}$ \\ ${ }^{1}$ V. I. Ilichev Pacific Oceanological Institute, Vladivostok, Russia \\ ${ }^{2}$ Vladivostok State University of Economics and Service, Vladivostok, Russia \\ Correspondence to: D. V. Stepanov (step-nov@ poi.dvo.ru)
}

Received: 17 January 2013 - Revised: 8 May 2013 - Accepted: 8 May 2013 - Published: 7 June 2013

\begin{abstract}
Observational evidence is presented for interaction between nonlinear internal Kelvin waves at the $\omega_{t, i}$ (where the $\omega_{t}$ is the semidiurnal frequency and the $\omega_{i}$ is the inertial frequency) and random oscillations of the background coastal current at the sub-inertial $\Omega$ frequency in the Japan/East Sea. Enhanced coastal currents at the sum $\omega_{+}$ and difference $\omega$-frequencies $\omega_{ \pm}=\omega_{t, i} \pm \Omega$ have properties of propagating Kelvin waves, which suggests permanent energy exchange from the sub-inertial band to the mesoscale $\omega_{ \pm}$band. This interaction may be responsible for a greaterthan-predicted intensification, steepening and breaking of boundary-trapped Kelvin waves. The problem of interaction between the nonlinear Kelvin wave at the frequency $\omega$ and the low-frequency narrowband noise with representative frequency $\Omega \ll \omega$ is investigated using the theory of nonlinear weak dispersion waves.
\end{abstract}

\section{Introduction}

A significant portion of the oceanic energy in coastal zones concentrates into an internal wave field. Wind-derived mesoscale internal waves, sub-inertial random oscillations of hydrological fields and topographical internal wave generation are the major sources of the energy. Energy cascades from low to high frequency in internal waves and then to turbulent dissipation associated with the nonlinearity of the internal wave field. Though the nonlinearity is believed to be a major factor resulting in significant changes of the internal wave spectrum, nonlinear processes and their parameterization remain important problems in physical oceanography.

Studies (Filonov and Novotryasov, 2005, 2007; Novotryasov et al., 2011) have shown that the nonlin- ear distortion of nondispersive wave profile generates a large number of tidal wave harmonics that propagate synchronously. More complex processes arise from the interaction of waves at odd frequencies. A nondispersive wave field with collinear wave propagation undergoes a cascade process when waves at the frequency $\omega_{1}$ and $\omega_{3}$ generate new waves at the frequency $\omega_{4}=\omega_{3}+\omega_{1}$, and so on. These processes result in a nonlinear decay of the internal wave field energy.

In this paper, we present observational evidence for interactions among narrowband sub-inertial random oscillations of the background coastal current at the frequency $\Omega$ and nonlinear internal Kelvin waves at the frequency $\omega_{0}$ (semidiurnal $M_{2}$ or inertial $f$ ), as well as its harmonics. Our findings are based on significant spectral peaks at the sum $\Omega+\omega_{0}$ and difference $\Omega-\omega_{0}$ frequencies surrounding the carrier frequency $\omega_{0}$ and sum $\Omega+n \omega_{0}$ and difference $\Omega-n \omega_{0}$ frequencies surrounding its harmonics. Isotherm displacement and kinetic energy spectra were obtained in the coastal zone of the Japan/East Sea. To describe the interactions, we used a model based on the Hopf equation (Gurbatov et al., 1990).

This paper is organized as follows: Sect. 2 describes the experiments performed in the coastal zone of the Japan/East Sea. Section 3 discusses the experimental results for the nonlinear aspects of internal Kelvin waves in the coastal zone within the framework of the Hopf equation. Our conclusions are presented in Sect. 4.

\section{Internal Kelvin wave observations}

For several years, we conducted internal wave experiments near the Gamov peninsula in the coastal zone of the Japan/East Sea, which is shown in Fig. 1. 

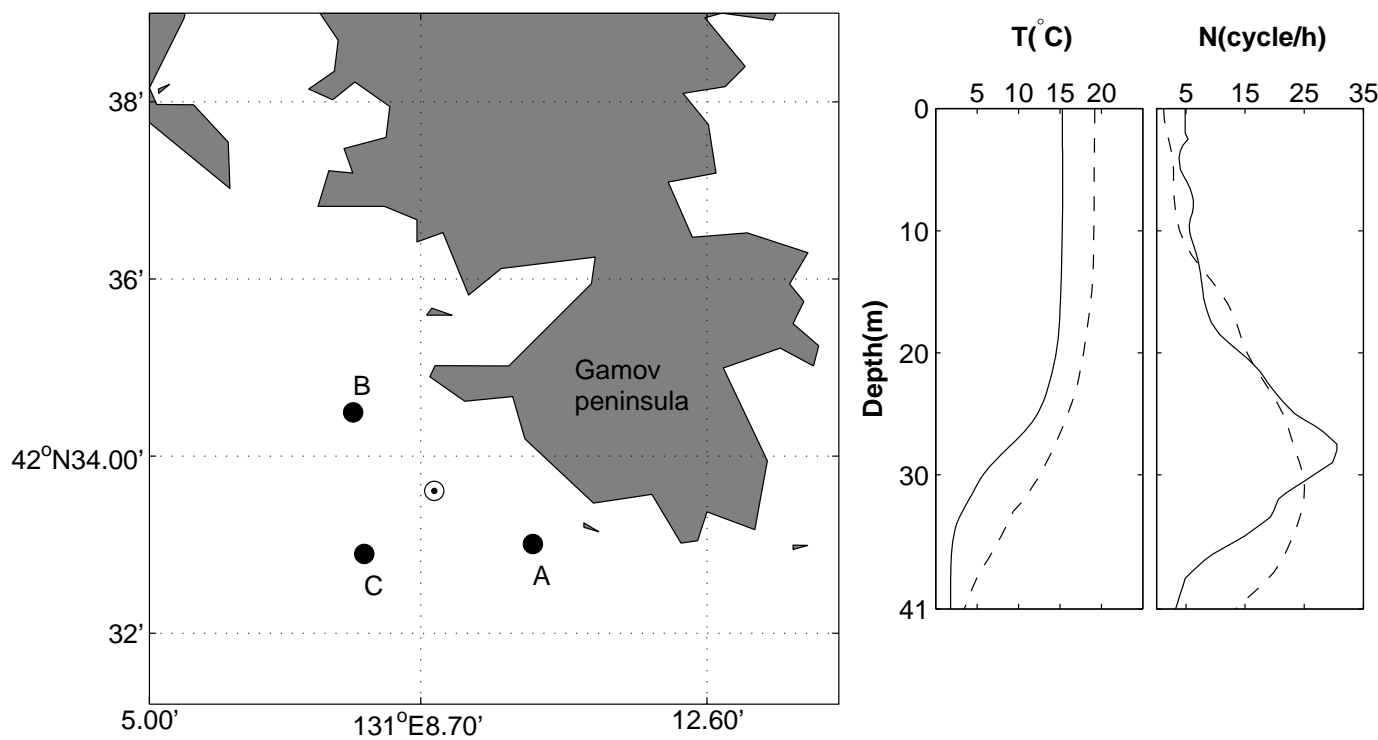

Fig. 1. (Left panel) Study area on the shelf of the Japan/East Sea in 1999, 2004 and 2012. The A and B mooring locations in September 2004 are shown, and the A, B and C mooring locations in September 2012 are shown. The circle indicates the location of the mooring vessel from which hourly casts were conducted in the autumn of 1999, 2004 and 2012. (Right panel) The vertical profiles of the background temperature $T$ and buoyancy frequency $N$ are shown in September 2004 (dashed line) and 2012 (solid line).

In the first experiment, we collected current meter records in the autumn of 1999 from a mooring deployed at $\left(42^{\circ} \mathrm{N}\right.$ $34^{\prime}, 131^{\circ} \mathrm{E} 09^{\prime}$ ) (indicated by the circle in Fig. 1). To gauge the temperature, speed and direction of the currents, the buoy was equipped with a Russian-made POTOK integral instrument at a depth of $35 \mathrm{~m}$. The POTOK has a temperature measurement resolution of $\pm 0.05^{\circ} \mathrm{C}$. The sampling rate was $15 \mathrm{~min}$. Vertical temperature and salinity profiles were collected from 12 to 13 September from a vessel anchored near the mooring using the Canadian Guideline CTD profiler. The profiling errors were no greater than $0.01{ }^{\circ} \mathrm{C}$ for temperature and 0.02 psu for salinity. A total of 25 hourly casts were made.

In the second experiment, internal wave band temperature fluctuations were measured over 18 days starting on 3 September 2004. The temperature time series were collected from the A and B moorings deployed along the coastline $800 \mathrm{~m}$ offshore and separated by a distance of $5.5 \mathrm{~km}$ (see Fig. 1). The moorings were equipped with digital thermographs, which have a measuring precision of $\pm 0.05{ }^{\circ} \mathrm{C}$ for temperature. The thermographs on the $\mathrm{A}$ and $\mathrm{B}$ moorings were deployed at depths of $28 \mathrm{~m}$ and $35 \mathrm{~m}$, respectively. The sampling rate was once per minute. The temperature and salinity vertical profiles were collected from a vessel anchored between the moorings using the Canadian Guideline CTD profiler from 20 to 21 September 2004. A total of 25 hourly casts were made. The details of this experiment (results, analytical methods, etc.) were reported by Novotryasov et al. (2005).
The data were analyzed using unified standard spectral techniques (Emery and Thomson, 1997). This approach involved (i) eliminating the low-frequency components using Tukey's cosine filter, (ii) splitting the resulting series into nine 37.2-hour segments (three semidiurnal tidal periods each), (iii) calculating and averaging the spectral densities by segments, and (iv) smoothing the averaged spectral densities using a five-point Tukey filter. There were approximately 10 degrees of freedom for processing the first experiment and 20 for the second, which provided reasonable reliability for the results of this spectral analysis.

Let us turn to the current velocity records. Figure 2a shows the time series smoothed using a Tukey window with a width of $1 \mathrm{~h}$ for the meridional (solid line) and zonal (bold line) components of the velocity in the coastal zone of the Japan/East Sea in the autumn of 1999. Note the lowfrequency component of variation in the time series. The spectrum of the clockwise rotary (CWR) component of the velocity is $S_{-}(\omega)=\left(S_{u u}+S_{v v}-2 Q_{u v}\right) / 8$, where $S_{u u}$ and $S_{v v}$ are the one-sided autospectra of the $u$ and $v$ Cartesian components of the velocity and $Q_{u v}$ is the quadrature spectrum between these two components. These spectra with $\mathrm{N}$ degrees of freedom $(\mathrm{N} \geq 10)$ were determined using unified statistical spectral techniques (Emery and Thomson, 1997).

Fig. 2b shows the time series of temperature fluctuations on the A and B moorings in September 2004, which were smoothed using a Tukey window with a width of $1 \mathrm{~h}$. The fluctuations have a quasi-periodic oscillation with an average period of approximately $18 \mathrm{~h}$. Cross-spectral analysis of the time series shows a phase difference between the fluctuations 

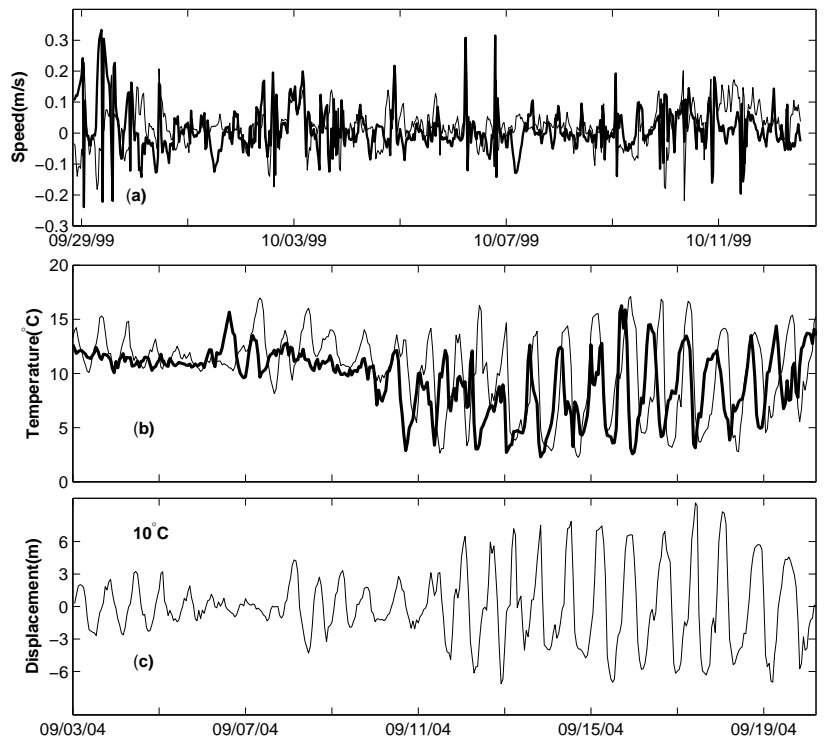

Fig. 2. (a) Time series of the meridional (solid line) and zonal (bold line) components of the current at a depth of $35 \mathrm{~m}$ smoothed using a Tukey window with a width of $1 \mathrm{~h}$ in the autumn of 1999. (b) Time series of the temperature variations from the A mooring (bold line) and B mooring (solid line) smoothed using a Tukey window with a width of $1 \mathrm{~h}$ in September 2004. (c) Time series of the isotherm $\left(10^{\circ} \mathrm{C}\right)$ displacements from the B mooring in September 2004.

at an inertial frequency of approximately $4.1 \mathrm{~h}$ and a mean propagation velocity between the moorings of approximately $0.38 \mathrm{~m} \mathrm{~s}^{-1}$. The phase velocity of the first baroclinic Kelvin mode with a frequency of $1 / 18$ cycle $^{-1}$ is close to $0.4 \mathrm{~m} \mathrm{~s}^{-1}$. Figure 1 shows the background temperature and buoyancy frequency profiles for the study site. Figure $2 \mathrm{c}$ shows the time series of the isotherm $\left(10^{\circ} \mathrm{C}\right)$ displacements calculated by $\zeta=\delta T\left(\frac{d<T>}{d z}\right)^{-1}$, where $\delta T$ is the temperature variations at a depth of $35 \mathrm{~m}$ and $<T>$ is the mean temperature profile in September 2004 . Analysis of the isotherm $\left(10^{\circ} \mathrm{C}\right)$ displacements shows that the ratio of the mean isotherm displacement to full water depth is approximately 0.3 relative units. Nonlinearity will thus influence the propagation of Kelvin waves.

In September 2012, we conducted an experiment that confirmed the existence of internal Kelvin waves in this coastal zone. The background temperature and buoyancy frequency profiles indicate the presence of an upper mixed layer $(2 / 3$ of the full water depth, $H$ ) and a stratified bottom layer characterized as having the background temperature gradient of $\frac{d<T>}{d z}=1{ }^{\circ} \mathrm{Cm}^{-1}$ and a maximal buoyancy frequency value at a depth of $30 \mathrm{~m}$ (see Fig. 1). Figure 3a shows the time series of temperature smoothed using a Tukey window with a width of $1 \mathrm{~h}$ from the $\mathrm{A}$ and $\mathrm{C}$ moorings at a depth of $30 \mathrm{~m}$. There were two stages of temperature variability during the experiment. The first stage was characterized by a mean period of approximately $14 \mathrm{~h}$, standard deviations of $\sigma_{A}$ and $\sigma_{B} \approx 2.9^{\circ} \mathrm{C}$ and $\sigma_{C} \approx 2.2^{\circ} \mathrm{C}$ on the $\mathrm{A}, \mathrm{B}$ and $\mathrm{C}$ moorings,

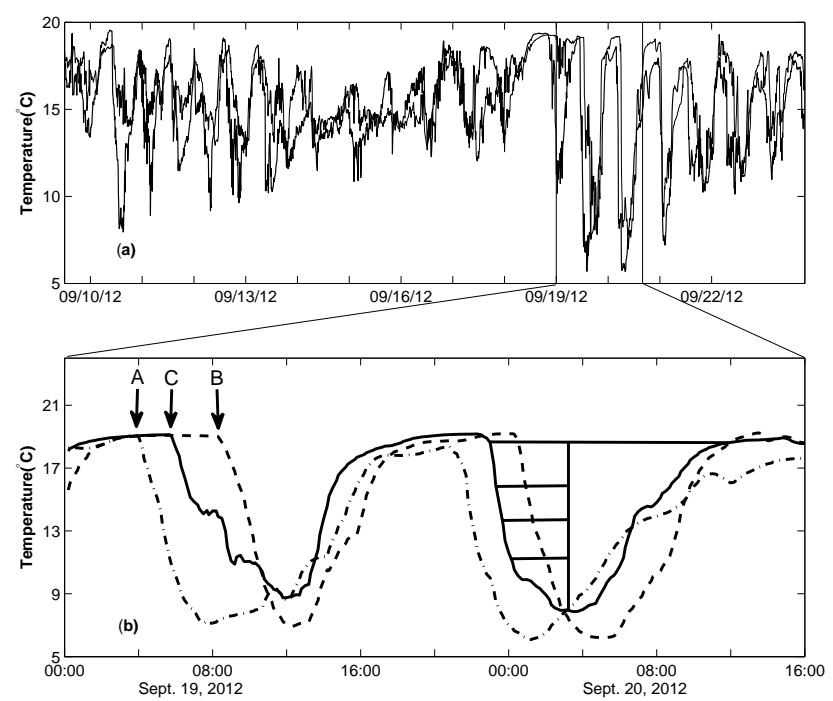

Fig. 3. (a) Time series of the temperature variations from the A mooring (bold curve) and B mooring (light curve) smoothed using a Tukey window with a width of $1 / 3 \mathrm{~h}$ are shown in September 2012. (b) Time series of the temperature variations from the A mooring (dash-dot line), B mooring (dashed line) and C mooring (solid line) smoothed using a Tukey window with a width of $3 \mathrm{~h}$ are shown during the temperature front propagation in September 2012. Asymmetry between the phase of the temperature increase and that of the temperature decrease is clearly shown.

respectively, and a duration of 9 days. At the end of the ninth day, the A mooring registered the presence of a sharp temperature front with a steepness of $(13 / 12){ }^{\circ} \mathrm{Cm}^{-1}$; this was the origin of the second stage. Figure $3 \mathrm{~b}$ shows the temperature records from the $\mathrm{A}, \mathrm{B}$ and $\mathrm{C}$ moorings during the front propagation. After passage of the wave front, the temperature variations had an asymmetric quasi-harmonic form with a mean period of $18 \mathrm{~h}$ and standard deviations of $\sigma_{A}$ and $\sigma_{B} \approx 3.1{ }^{\circ} \mathrm{C}$ and $\sigma_{C} \approx 2.7^{\circ} \mathrm{C}$ on the $\mathrm{A}, \mathrm{B}$ and $\mathrm{C}$ moorings, respectively.

With these data we calculated the mean velocities of the temperature maxima and minima on the A-B track during different stages of the experiment. During the first stage of the experiment, the mean propagation velocities of the temperature maxima and minima were $\left\langle c_{+1}>\approx 0.42 \mathrm{~m} \mathrm{~s}^{-1}\right.$ and $\left.<c_{-1}\right\rangle \approx 0.36 \mathrm{~m} \mathrm{~s}^{-1}$, respectively. During the second stage, these velocities were $\left\langle c_{+2}>\approx 0.35 \mathrm{~m} \mathrm{~s}^{-1}\right.$ and $\left\langle c_{-2}>\approx\right.$ $0.26 \mathrm{~m} \mathrm{~s}^{-1}$, respectively. Thus, we observed two types of temperature disturbances in the Japan/East Sea coastal zone in September 2012. The first type had a period of $14 \mathrm{~h}$ and a mean propagation velocity of $\left\langle c_{1}>\approx 0.39 \mathrm{~m} \mathrm{~s}^{-1}\right.$. The second type had a period of $18 \mathrm{~h}$ and a mean propagation velocity of $\left\langle c_{2}\right\rangle \approx 0.31 \mathrm{~m} \mathrm{~s}^{-1}$. These temperature disturbances had an asymmetric form and significant amplitudes, which decreased exponentially with increasing distance across the coast as $\exp (-y / L)$, where $L=D \ln \left(\sigma_{A} / \sigma_{C}\right)$ and $D$ is distance from the $\mathrm{C}$ mooring to the wave paths. 


\section{Theoretical description of observational Kelvin waves}

We used a weak nonlinear Kelvin wave model to interpret the observed temperature variability in the coastal zone of the Japan/East Sea. According to the model, a linear relationship exists between the temperature variation $\delta T$ and the isotherm displacement $\zeta$ as follows: $\delta T=\frac{d<T>}{d z} \zeta$. Therefore, temperature variations can be considered as an indicator of the vertical displacement of fluid particles in the layer with the linear temperature profile.

Let us consider the propagation of coastal internal Kelvin waves (IKWs) in a layer with depth $H$ of a continuously stratified fluid with the buoyancy frequency $N(z)$. In this case we could represent the solution for vertical displacement of the pycnocline $\eta$ by an expansion in eigenmodes (Reznik, 2002; Ostrovsky, 1978):

$\eta(x, y, z, t)=\sum_{m} a_{m} \eta_{m}(x, t) e^{\left(-y f / c_{m}\right)} \phi_{m}(z)$.

Here $y$ is the transverse coordinate, $a_{m}$ constants, $f$ the inertial frequency, and $\phi_{m}(z)$ the orthogonal eigenfunctions that satisfy the eigenvalue problem:

$\frac{\mathrm{d}^{2} \phi_{m}}{\mathrm{~d} z^{2}}+\frac{N^{2}(z)}{c_{m}^{2}} \phi_{m}=0$,

$\phi_{m}(0)=\phi_{m}(-H)=0$,

$\phi_{\max }=1$,

where $c_{m}$ are the eigenvalues, which have the meaning of a long-wave velocity for each linear IKW mode with number $m$. In the general case, after solving Eqs. (2)-(4), approximate equations describing the dependence of physical values of long IKW at the frequencies $\omega_{0} \ll N_{\max }$ on $x$ and $t$ can be derived with the use of different perturbation schemes. Here, we used a rather general model suggested by Ostrovsky (1978). At small nonlinearity with no resonant coupling between different modes, the vertical displacement for the first most powerful baroclinic mode can be shown to obey the simple wave equation

$\frac{\partial \eta_{1}}{\partial t}+c_{1}\left(1+\frac{3}{2} \frac{\alpha_{1} \eta_{1}}{H}\right) \frac{\partial \eta_{1}}{\partial x}=0$,

where $\alpha_{1}$ is the parameter describing nonlinearity that is determined by

$\alpha_{1}=\left[H \int_{-H}^{0}\left(\frac{\mathrm{d} \phi_{1}}{\mathrm{~d} z}\right)^{3} \mathrm{~d} z\right] /\left[\int_{-H}^{0}\left(\frac{\mathrm{d} \phi_{1}}{\mathrm{~d} z}\right)^{2} \mathrm{~d} z\right]$.

Equation (5) is solvable as an initial Cauchy problem. However, in our experiments, we are addressing time series of measurements of temperature and current velocity at some fixed point $x=\hat{x}$, and we want to predict the spatial development of the wave as it propagates along the boundary of the coastal area, given that it is known as a function of time at the coastal area entrance. We thus come to consider a boundary Cauchy problem for the simple wave equation (Osborne, 1995). After substituting for $\tau=t-x c_{1}^{-1}, X=\epsilon x$, where $\epsilon \ll 1$ and $U \approx c_{1} \eta_{1} H^{-1}$ is the alongshore velocity, Eq. (5) is reduced to the Hopf equation

$\frac{\partial U}{\partial X}-\beta U \frac{\partial U}{\partial \tau}=0$,

$U(X=0, t)=U_{0}(t)$.

Here, $\beta=(3 / 2) \alpha_{1} / c_{1}^{2}$ and the further index 1 is omitted. This equation is first-order accurate for wave amplitude $\epsilon=$ $a_{0} / H$, long waves $H / \lambda \ll 1$ and low-frequency $\omega / N_{\max } \ll$ 1 internal waves.

We calculated the phase speed of the IKW first mode $c_{p}=$ $0.45 \mathrm{~m} \mathrm{~s}^{-1}$ as solutions of the boundary value problem in Eq. (2) with the buoyancy frequency profile $N(z)$ (see Fig. 1) and then compared it with the propagation velocities of the observed temperature disturbances $\left(<c_{1}\right\rangle \approx 0.39 \mathrm{~m} \mathrm{~s}^{-1}$ and $<c_{2}>\approx 0.31 \mathrm{~m} \mathrm{~s}^{-1}$ ). It has been suggested that one of the reasons for the differences between velocities is the interaction of the IKW with the background flow. The IKW propagating against the background flow generates a temperature disturbance with a frequency of $\omega_{M}=\omega_{0}\left(1+U_{b} / c_{p}\right)^{-1}$, where $U_{b}$ is the speed of the background flow and $\omega_{0}$ the frequency of the IKW at the $U_{b}=0$ (White, 1972). Therefore, the propagation velocity of the temperature disturbance on the track is

$c_{M}=c_{p}\left(T_{0} / T_{M}\right)$,

where $T_{M}$ is the period of the temperature disturbances registered by the A, B and C moorings and $T_{0}=2 \pi / \omega_{0}$. It has been established (Navrotsky et al., 2004; Novotryasov et al., 2005) that the strong variability in temperature is derived from the semidiurnal oscillations having a frequency $\omega_{0}=2 \pi / 12.4 \mathrm{~h}^{-1}$ in the coastal zone of the Japan/East Sea. Therefore, we suggest that the major source of the temperature perturbations is the internal wave with the frequency $\omega_{0}$. In this case, according to Eq. (9), the propagation velocities of the temperature disturbances during the first and second stages of the experiment must be closed by the relation $c_{2}=(14 / 18) c_{1}$. Considering that $c_{1}=0.39 \mathrm{~m} \mathrm{~s}^{-1}$, we find that $c_{2}=0.31 \mathrm{~m} \mathrm{~s}^{-1}$. This finding is consistent with the propagation velocity of the temperature disturbances during the second stage of the experiment $\left\langle c_{2}>\approx 0.31 \mathrm{~m} \mathrm{~s}^{-1}\right.$.

In accordance with the weak nonlinear IKW model, the wave phase velocity of the IKW along the track was related to the wave amplitude at the $\mathrm{A}$ and $\mathrm{C}$ moorings by

$c_{M}=f_{0} D \ln \left(\zeta_{A} / \zeta_{B}\right)$, 
where $f_{0}$ is the Coriolis parameter. Using Eq. (10) and the data from the second stage of the experiment (i.e., where $D \approx 2200 \mathrm{~m}, \sigma_{A} \approx 3.1{ }^{\circ} \mathrm{C}$, and $\left.\zeta_{A} / \zeta_{B} \approx \sigma_{A} / \sigma_{B}\right)$, we calculated the phase velocity of the coastal Kelvin wave $c_{2}=0.29$ $\mathrm{ms}^{-1}$, which was close to the value of the mean propagation velocity of the temperature disturbance during the second stage of the experiment $\left\langle c_{2}>\approx 0.31 \mathrm{~m} \mathrm{~s}^{-1}\right.$.

Based on the model of weak nonlinear IKW, we suggest that the difference between the propagation velocities of the temperature maxima and minima results from the nonlinearity of the internal Kelvin wave field. The solutions to Eqs. (7) and (8) yield a simple wave (Osborne, 1995), which depends on the fluid particles' vertical displacement as follows:

$V=c_{p}(1+\alpha \zeta / H)$

Therefore, the wave crest speed differs from the wave trough speed. From Eq. (11), we obtain the difference between the wave crest and wave trough speeds as $\delta V=2 c_{p} \alpha \zeta H^{-1}$. If $\alpha>0$, then the wave crest propagates faster than the wave trough, and the wave profile becomes asymmetric.

Using Eq. (11) with parameters $\zeta_{1} \approx 2 \mathrm{~m}, \alpha=1.3$ and $H \approx 41 \mathrm{~m}$, we calculated $\delta V=0.055 \mathrm{~m} \mathrm{~s}^{-1}$, which is close to $\delta V_{1}=0.06 \mathrm{~m} \mathrm{~s}^{-1}$ obtained from the observational data. It is easy to show that this relationship also holds for the second stage of the experiment. We therefore showed that the weak nonlinear IKW model can be used to interpret the observed temperature variability in the coastal zone of the Japan/East Sea.

Let us now show that the weak nonlinear IKW model can be used to interpret the observational flow velocity in the coastal zone of the Japan/East Sea.

We consider the interaction of a nonlinear IKW with the frequency $\omega_{0}$ (semidiurnal $\omega_{t}$ or inertial $\omega_{i}$ ) and narrowband sub-inertial noise using the nonlinear internal wave model described by Eq. (7). Let the alongshore velocity $U(X, \tau)$ at the boundary of the coastal area $X=0$ be the superposition of the internal wave with frequency $\omega_{0}$, amplitude $A_{0}$ and noise $\vartheta(t)$ with a typical width $\gamma \ll \omega_{0}$ and variance $s^{2}$ :

$U(X=0, t)=U_{0}(t)=A_{0} \cos \left(\omega_{0} t+\varphi(t)\right)+\vartheta(t)$,

where $\varphi$ is the random phase with a uniform distribution in the interval $[-\pi,+\pi]$.

We confine our analysis to the wave evolution stage, which is characterized by the following condition: $X<X_{T}$, where $X_{T}=\left(\beta A_{0} \omega_{0}\right)^{-1}$. In this stage, the steepening of the IKW appears, but it is not accompanied by the generation of internal solitons. We introduce parameter $d_{T}=X / X_{T}$, which determines the similarity between a Kelvin shock-wave and the linear IKW, and then consider the case $d_{T}<1$. For this case, the spectral density of the wave field $U$, according to Gurbatov et al. (1990) and Filonov and Novotryasov (2007), is

$$
\begin{array}{r}
\Phi(X, \omega)=-\frac{J_{0}\left(\omega d_{T} / \omega_{0}\right)}{2 \pi i \omega \beta X} \times \\
\times \int_{-\infty}^{+\infty}\left\{e^{-i \omega \beta X \vartheta(\tau)}-1\right\} e^{i \omega \tau} d \tau-\sum_{\substack{n=-\infty \\
n \neq 0}}^{\infty} \frac{i^{n} J_{n}\left(\omega d_{T} / \omega_{0}\right)}{2 \pi i \omega \beta X} \times \\
\times \int_{-\infty}^{+\infty}\left\{e^{(i n \varphi-i \omega \beta X \vartheta(\tau))}-1\right\} e^{i\left(\omega-n \omega_{0}\right) \tau} d \tau .
\end{array}
$$

From Eq. (13), we can affirm that the first term is the spectral density of the sub-inertial oscillations and the second term is the spectral density of the IKW distorted by the sub-inertial oscillations. We consider the spectral density of the IKW near the harmonics with the number $n$ of the carrier frequency $\omega_{0}$. Because $\gamma \ll \omega_{0}$, we can replace $\omega$ by $n \omega_{0}$ in the exponential rate of Eq. (13). From the inverse Fourier transformation of Eq. (13), we obtain the equation for the harmonic with number $n$ :

$U_{n}(X, \tau)=A_{n}(X) \cos \left[n \omega_{0} \tau+n\left(\varphi(\tau)-\omega_{0} \beta X \vartheta(\tau)\right)\right]$,

and the amplitude depending on parameter $d_{T}=A_{0} \beta \omega_{0} X$ is

$A_{n}(X)=A_{0} \frac{2 J_{n}\left(n d_{T}\right)}{n d_{T}}$.

We performed a more detailed spectral analysis of the nonlinear IKWs when the sub-inertial noise consists of both regular and stochastic components: $\vartheta(t)=\vartheta_{0} \cos (\Omega t)+\vartheta_{S N}(t)$. Let the amplitude of the phase modulation related to the regular component $M=\beta \vartheta_{0} n \Omega X$ be small, i.e., $M \ll 1$. Additionally, let the IKWs have a narrowband spectrum, and for the subinertial noise width $\gamma \ll \omega_{0}$, let the amplitude and phase have Gaussian distributions. In this case, the correlation function of the harmonic with number $n$, taking into account Eqs. (14) and (15), is

$$
\begin{array}{r}
B_{n}(X, \tau)=\left(\frac { A _ { n } ^ { 2 } } { 2 } \left(\cos n \omega_{0} \tau+\frac{\left(\vartheta_{0} \beta n \omega_{0} X\right)^{2}}{2} \times\right.\right. \\
\left.\left.\times\left(\cos \left(\left(n \omega_{0}+\Omega\right) \tau\right)+\cos \left(\left(n \omega_{0}-\Omega\right) \tau\right)\right)\right)\right) e^{-n^{2} D_{\psi}(\tau) / 2} .
\end{array}
$$

Here, $D_{\psi}(\tau)=D_{\varphi}(\tau)+\left(\beta \omega_{0} X\right)^{2}\left(s_{S N}^{2}-B_{S N}(\tau)\right), D_{\varphi}$ is the structure function of the IKW phase, and $s_{S N}^{2}$ and $B_{S N}(\tau)=<\vartheta_{S N}(t+\tau) \vartheta_{S N}(t)>$ are the dispersion and correlation function of the subinertial noise, respectively. We performed a Fourier transform of the correlation function $B_{n}(X, \tau)$ and obtained the formula for the spectrum of the harmonic $n$ :

$$
\begin{gathered}
S_{n}(X ; \omega)=\frac{A_{n}^{2}}{2} \tilde{S}_{n}\left(\omega-n \omega_{0}\right)+\frac{\left(A_{n} \vartheta_{0} \beta n \omega_{0} X\right)^{2}}{4} \times \\
\quad \times\left(\tilde{S}_{n}\left(\omega-\left(n \omega_{0}+\Omega\right)\right)+\tilde{S}_{n}\left(\omega-\left(n \omega_{0}-\Omega\right)\right)\right),
\end{gathered}
$$



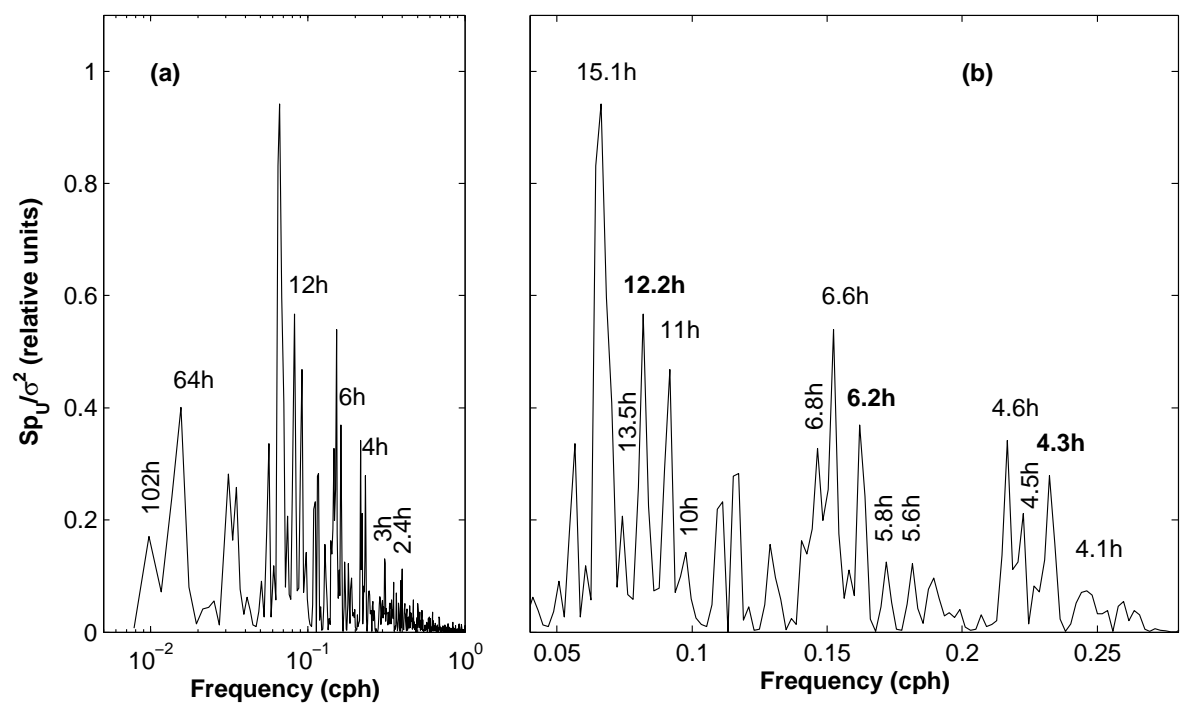

Fig. 4. (a) Normalized clockwise rotary spectrum of current versus $\log \omega$ for currents measured at a depth of $35 \mathrm{~m}$ in the autumn of 1999 . Numbers over the peaks are their periods. (b) The increased fragment of the spectrum surrounding the semidiurnal frequency. The bold numbers 12.2, 6.2 and 4.3 are the carrier periods.

Table 1. Periods of the peaks of the clockwise rotary spectrum (bold) and the calculated periods in hours (italic).

\begin{tabular}{cccccccccc}
\hline $1 / \Omega_{1,2}$ & $1 / \omega_{+}$ & $1 / \omega_{t}(h)$ & $1 / \omega_{-}$ & $1 / \omega_{+}$ & $1 /\left(2 \omega_{t}\right)$ & $1 / \omega_{-}$ & $1 / \omega_{+}$ & $1 /\left(3 \omega_{t}\right)$ & $1 / \omega_{-}$ \\
\hline $\mathbf{6 4}$ & 10.2 & $\mathbf{1 2 . 2}$ & 15.1 & 5.7 & $\mathbf{6 . 2}$ & 6.9 & 4.0 & $\mathbf{4 . 3}$ & 4.6 \\
$\mathbf{6 4}$ & $\mathbf{1 0}$ & $\mathbf{1 2 . 2}$ & $\mathbf{1 5 . 1}$ & $\mathbf{5 . 6}$ & $\mathbf{6 . 2}$ & $\mathbf{6 . 8}$ & $\mathbf{4 . 1}$ & $\mathbf{4 . 3}$ & $\mathbf{4 . 6}$ \\
$\mathbf{1 0 2}$ & 10.9 & $\mathbf{1 2 . 2}$ & 13.9 & 5.8 & $\mathbf{6 . 2}$ & 6.6 & 4.1 & $\mathbf{4 . 3}$ & 4.5 \\
$\mathbf{1 0 2}$ & $\mathbf{1 1}$ & $\mathbf{1 2 . 2}$ & $\mathbf{1 3 . 5}$ & $\mathbf{5 . 8}$ & $\mathbf{6 . 2}$ & $\mathbf{6 . 6}$ & $\mathbf{4 . 1}$ & $\mathbf{4 . 3}$ & $\mathbf{4 . 5}$ \\
\hline
\end{tabular}

where

$\tilde{S}_{n}(X ; \omega)=\frac{1}{2 \pi} \int_{-\infty}^{+\infty} \exp \left[-n^{2} D_{\psi}(\tau) / 2\right] \cos (\omega \tau) d \tau$.

From Eq. (17), we can affirm that the spectrum of the $U(X, \tau)$ has harmonics at the frequencies $n \omega_{0}$ as well as harmonics with sum and difference frequencies $\omega_{ \pm}=n \omega_{0} \pm \Omega$. From Eq. (15), the harmonic amplitudes increase with the distance traversed by the wave. This means that interaction between nonlinear IKWs and sub-inertial noise occurs at the coastal zone, which is accompanied by the intensification of IKWs with side frequencies $\omega_{ \pm}=n \omega_{0} \pm \Omega$.

Let us turn to the current velocity records. Figure $4 \mathrm{a}$ shows the CWR spectrum of currents versus $\log \omega$ normalized by the maximal value. There are two peaks at the representative frequencies $\Omega_{1} \approx 1 / 64 \mathrm{cph}$ and $\Omega_{2} \approx 1 / 102 \mathrm{cph}$ in the low-frequency band of the spectrum. Groups of well-defined significant spectral peaks surrounding the semidiurnal frequency $\omega_{t} \approx 1 / 12.4 \mathrm{cph}$ and the $1-4$ harmonics are observed. Our estimations show that the magnitudes of these spectral peaks and the number of harmonics are interrelated by the approximate relation

$A_{12}: A_{6}: A_{4}: A_{3}: A_{2.4} \approx 1: 2: 3: 4: 5$

(with error $<20 \%$ ).

Figure $4 \mathrm{~b}$ shows the increased fragment of the spectrum surrounding the semidiurnal frequency. Note the significant well-defined spectral peak at the $\omega_{t}=1 / 12.2 \mathrm{cph}$ frequency surrounded by side peaks. In Table 1 , we present the periods (bold numbers) of the spectral peaks of the CWR spectrum (see Fig. 4b); the periods (italic numbers) are calculated using the formula $1 / \omega_{ \pm}=1 /\left(n \omega_{t} \pm \Omega_{1,2}\right)$, where $\Omega_{1,2} \approx 1 / 64,1 / 102 \mathrm{cph}$ and $n=1,2,3$. Table 1 shows that the maximum relative error between the period of the spectral peaks and the periods calculated using the formula $1 / \omega_{ \pm}=$ $1 /\left(n \omega_{t} \pm \Omega_{1,2}\right)$ can reach a value of approximately $6 \%$ at the frequency $1 / 10.8 \mathrm{cph}$. The spectral analysis of the clockwise rotary velocity thus shows that the spectrum is determined by oscillations with the tidal frequency $\omega_{t} \mathrm{cph}$ and the $\Omega_{1,2} \mathrm{cph}$ frequencies from the sub-inertial band. The spectrum also exhibits a fine structure in the neighborhood of the frequency $\omega_{t}$, with side peaks at the frequencies determined by

$\omega_{ \pm}=n \omega_{t} \pm \Omega_{1,2}$ 

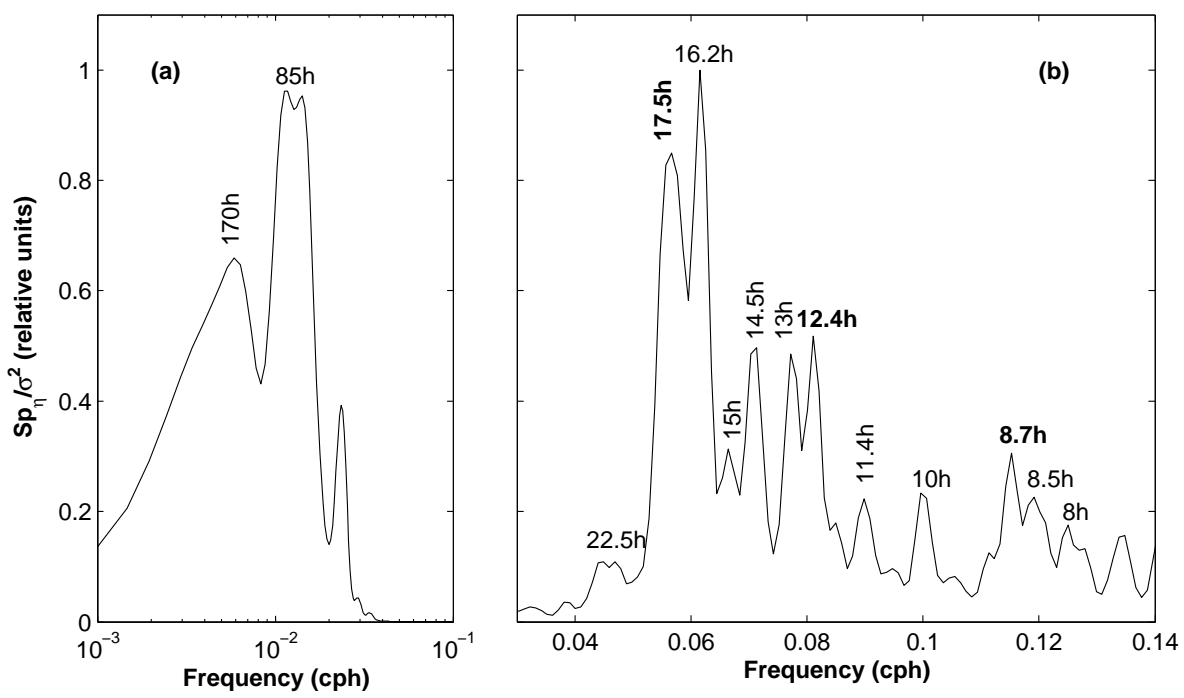

Fig. 5. (a) Normalized averaged spectrum of the isotherm $\left(10^{\circ} \mathrm{C}\right)$ vertical displacements versus $\log \omega$ from the A and B moorings in September 2004. Numbers over the peaks are their periods. (b) Increased fragment of the spectrum surrounding the inertial and semidiurnal frequencies. The bold numbers $\mathbf{1 7 . 5}, \mathbf{1 2 . 4}$ and $\mathbf{8 . 7}$ are the carrier periods.

Table 2. Periods of the peaks of the isotherm displacement spectrum (bold) and the calculated periods in hours (italic).

\begin{tabular}{cccccccccc}
\hline $1 / \Omega_{1,2}$ & $1 / \omega_{+}$ & $1 / \omega_{t}$ & $1 / \omega_{-}$ & $1 / \omega_{+}$ & $1 / \omega_{i}$ & $1 / \omega_{-}$ & $1 / \omega_{+}$ & $1 /\left(2 \omega_{i}\right)$ & $1 / \omega_{-}$ \\
\hline $\mathbf{8 5}$ & 10.8 & $\mathbf{1 2 . 4}$ & 14.5 & 14.5 & $\mathbf{1 7 . 5}$ & 22.0 & 7.9 & $\mathbf{8 . 7}$ & 9.7 \\
$\mathbf{8 5}$ & $\mathbf{1 1 . 4}$ & $\mathbf{1 2 . 4}$ & $\mathbf{1 4 . 5}$ & $\mathbf{1 5 . 0}$ & $\mathbf{1 7 . 5}$ & $\mathbf{2 2 . 5}$ & $\mathbf{8 . 0}$ & $\mathbf{8 . 7}$ & $\mathbf{1 0 . 0}$ \\
$\mathbf{1 7 0}$ & 11.6 & $\mathbf{1 2 . 4}$ & 13.4 & 15.9 & $\mathbf{1 7 . 5}$ & 19.5 & 8.3 & $\mathbf{8 . 7}$ & 9.2 \\
$\mathbf{1 7 0}$ & $\mathbf{1 1 . 4}$ & $\mathbf{1 2 . 4}$ & $\mathbf{1 3 . 0}$ & $\mathbf{1 6 . 2}$ & $\mathbf{1 7 . 5}$ & - & $\mathbf{8 . 5}$ & $\mathbf{8 . 7}$ & - \\
\hline
\end{tabular}

A similar structure is observed in the spectrum around the first and second harmonics of the semidiurnal frequency $\omega_{t}$.

Figure 5 shows the averaged spectrum of the isotherm displacements normalized by the maximum value. Figure $5 \mathrm{a}$ shows increased fragment in the low-frequency band of the spectrum. There are two peaks at the representative frequencies $\Omega_{1} \approx 1 / 85 \mathrm{cph}$ and $\Omega_{2} \approx 1 / 170 \mathrm{cph}$. Figure $5 \mathrm{~b}$ shows increased fragment of the spectrum surrounding the inertial and semidiurnal frequencies. There are groups of significant spectral peaks at the inertial frequency and its first harmonic as well as the peaks surrounding the semidiurnal frequency.

Table 2 contains two types of numbers: the bold numbers are the periods of significant peaks of the isotherm $\left(10^{\circ} \mathrm{C}\right)$ displacement spectrum; the italicized numbers are the periods calculated by $1 / \omega_{ \pm}=1 /\left(n \omega_{t, i} \pm \Omega_{1,2}\right)$, where $\Omega_{1,2}=$ $1 / 85,1 / 170 \mathrm{cph}, \omega_{t}=1 / 12.4 \mathrm{cph}, n=1$ and $\omega_{i}=1 / 17.5$ cph and $n=1,2$.

As indicated by Tables 1 and 2, the periods of the spectral peaks (bold numbers) and periods (italic numbers) calculated by $1 / \omega_{ \pm}=1 /\left(n \omega_{t, i} \pm \Omega_{1,2}\right)$ have approximately equal values. Spectral analyses of the current meter, temperature and isotherm $\left(10^{\circ} \mathrm{C}\right)$ displacement records in the coastal zone of the Japan/East Sea show that the spectra are determined by oscillations with near-inertial and tidal frequencies, as well as by frequencies from the sub-inertial band. The spectra have fine structure in the neighborhood of the frequencies, with side peaks at frequencies determined by Eq. (19).

\section{Concluding remarks}

Internal Kelvin waves play an important role in the dynamics of the coastal oceans. The nonlinearity of the Kelvin waves results in interactions of the waves with each other, the generation of wave harmonics, and the waves at the combination frequencies. Randomness of the velocity field is a typical feature of coastal oceans. Developing models of the interaction of the internal wave field with a random velocity field is an important problem in physical oceanography.

In this paper, we presented a very simple model of the interaction of the narrowband sub-inertial noise with the nonlinear internal Kelvin wave field for describing all the interaction features in the coastal zone of the Japan/East Sea. For example, the model does not allow a space-time transformation of the internal wave field in the coastal zone. However, using the model, we attempted to show a possible basis for the significant finite-width spectral peaks surrounding semidiurnal and inertial frequencies, as well as their harmonics in 
the kinetic energy and isotherm displacement spectra in the coastal zone.

We have presented observational evidence for the interactions between sub-inertial random oscillations of the background current at the representative frequency $\Omega$ and nonlinear Kelvin waves at the semidiurnal $\omega_{t}$ or the inertial $\omega_{i}$ frequencies. Our findings were based on analyses of current meter and temperature records in the coastal zone of the Japan/East Sea. We have shown that the spectra are determined by oscillations with near-inertial and semidiurnal frequencies, as well as frequencies from the sub-inertial band. The isotherm displacement and wave current spectra have fine structure in the neighborhood of the semidiurnal and inertial frequencies with side peaks at frequencies determined by the formula $\omega_{ \pm}=\omega_{t, i} \pm \Omega$. Thus, the energy exchange occurs from the low-frequency band to the mesoscale band of internal Kelvin waves. Hence, as a result of the interaction, the energy of internal Kelvin waves in the coastal oceans can grow at the expense of the sub-inertial narrowband noise energy. Furthermore, as the first baroclinic Kelvin mode is the most powerful (see Fig. 1b), we expect intensification of the semidiurnal and inertial currents on the sub-surface and nearbottom layers of the coastal zone.

Acknowledgements. This work was supported by the ICMAT Severo Ochoa project SEV-2011-0087, the Russian Foundation for Basic Research Grant 12-05-00715-a and by the Presidential Grant No. MK-2962.2012.5.

Edited by: A. M. Mancho

Reviewed by: two anonymous referees

\section{References}

Emery, W. J. and Thomson, R. E.: Data Analysis Methods in Physical Oceanography, Pergamon Press, New York, 1997.

Filonov, A. and Novotryasov, V.: Features of the nonlinear wave spectrum in the coastal zone, Geophys. Res. Lett., 32, L15602, doi:10.1029/2005GL023046, 2005.

Filonov, A. and Novotryasov, V.: On a spectrum of nonlinear internal waves in the oceanic coastal zone, Nonlin. Processes Geophys., 14, 757-762, doi:10.5194/npg-14-757-2007, 2007.

Gurbatov, S. N., Malakhov, A. N., and Saichev, A. I.: Nonlinear random waves and turbulence in nondispersive media: waves, rays and particles, Manchester University Press, Manchester, 304 pp., 1991.

Müller, P., Holloway, G., Henyey, F., and Pomphrey, N.: Nonlinear interactions among internal gravity waves, Rev. Geophys., 24, 493-596, 1986.

Navrotsky, V. V., Lozovatsky, I. D., Pavlova, E. P., and Fernando, H. J. S.: Observations of internal waves and thermocline splitting near a shelf break of the Sea of Japan (East Sea), Cont. Shelf Res., 24, 1375-1395, 2004.

Novotryasov, V. V., Vanin, N. S., and Karnaukhov, A. A.: Manifestation of nonlinear properties of Kelvin internal waves in the coastal zone of the sea of Japan, Izv. Atmos. Ocean. Phys., 41, 611-619, 2005.

Novotryasov, V. V., Filonov, A., and Lavin, M. F.: Nonlinear internal tidal waves in a semi-enclosed sea (Gulf of California), Geophys. Res. Lett., 38, L24611, doi:10.1029/2011GL049886, 2011.

Osborne, A. R.: The inverse scattering transform: Tools for the nonlinear fourier analysis and filtering of ocean surface waves, Chaos, Solit. Fract., 5, 2623-2637, 1995.

Ostrovsky, L. A.: Nonlinear internal waves in a rotating ocean, Oceanology, 18, 119-125, 1978.

Reznik, G. M. and Grimshow, R.: Nonlinear geostrophic adjustment in the presence of a boundary, J. Fluid Mech., 471, 257-283, 2002.

White, W. B.: Doppler shift in the frequency of inertial waves observed in moored spectra, Deep-Sea Res., 19, 595-600, 1972.

Whitham, G. B.: Linear and Nonlinear Waves, John Wiley, New York, 1974. 